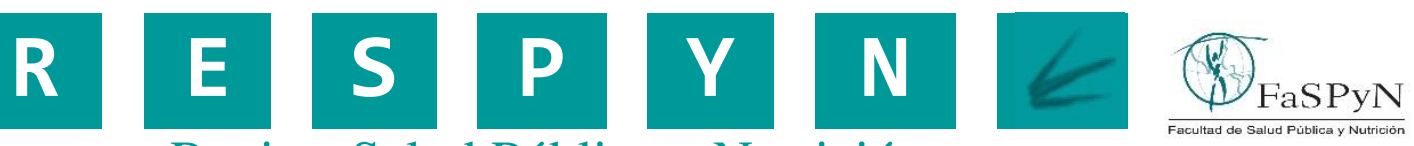 \\ Revista Salud Pública y Nutrición
}

\section{FACTORES ASOCIADOS AL DEBUT SEXUAL, ACTIVIDAD SEXUAL EN LÍNEA Y CALIFICACIÓN EN ESTUDIANTES DE MORELIA.}

\author{
FACTORS ASSOCIATED WITH SEXUAL DEBUT, SEXUAL ACTIVITY ONLINE AND GRADING IN MORELIA \\ STUDENTS.
}

\begin{abstract}
López Tapia Ricardo. ${ }^{1}$ Martínez Toledo José Luis. ${ }^{2}$
1 Facultad de Ciencias Médicas y Biológicas "Dr. Ignacio Chávez", México. 2 Hospital Infantil de Morelia "Eva Sámano de López Mateos", México.
\end{abstract}

Citation: López Tapia R., Martínez Toledo JL. (2018) Factores asociados al debut sexual, actividad sexual en línea y calificación en estudiantes de Morelia. Revista de Salud Pública y Nutrición, 17(1), 16-22.

Editor: Esteban G. Ramos Peña, Dr. CS., Universidad Autónoma de Nuevo León, Facultad de Salud Pública, Monterrey Nuevo León, México.

Copyright: (C2018 López Tapia R. et al. This is an open-access article distributed under the terms of Creative Commons Attribution License [CC BY 4.0], which permits unrestricted use, distribution, and reproduction in any medium, provided the original author and source are credited.

Competing interests: The authors have declared that no competing interests exist.

DOI: https://doi.org/10.29105/respyn17.1-3

Recibido: 9 de marzo 2018; $\quad$ Aceptado: 30 de abril 2018

Email: lopeztapiaricardo@hotmail.com 


\title{
FACTORES ASOCIADOS AL DEBUT SEXUAL, ACTIVIDAD SEXUAL EN LÍNEA Y CALIFICACIÓN EN ESTUDIANTES DE MORELIA.
}

\author{
López Tapia Ricardo. ${ }^{1}$ Martínez Toledo José Luis. ${ }^{2}$ \\ 1 Facultad de Ciencias Médicas y Biológicas "Dr. Ignacio Chávez". 2 Hospital Infantil de Morelia "Eva Sámano de López \\ Mateos".
}

\begin{abstract}
RESUMEN
Introducción: Las prácticas sexuales de los adolescentes se han convertido en un problema de salud pública a nivel mundial. Objetivo: Describir factores asociados al debut sexual en estudiantes, la actividad sexual en línea y sus calificaciones. Material y Métodos: Estudio observacional, transversal incluyó 673 estudiantes de secundarias y preparatorias públicas y privadas de Morelia. Se aplicó encuesta auto diligenciada sobre factores asociados a sexualidad. Resultados: $27.4 \%$ busca pornografía en internet una vez a la semana, ciber sexo lo practica $4.5 \%$, sexting $6.1 \%$. Internet resultó la principal fuente de información sexual (40.4\%). 20.1\% tuvo debut sexual, con media de 14.8 años (desviación estándar=1.7), 47.4\% lo hizo por curiosidad. Para el debut sexual resultaron significativos la disfunción familiar y tener pareja $(p=0.040$ y $p=0.000$ ), no tuvieron significancia el tipo de institución, religión y autoestima. Los estudiantes sin debut sexual tuvieron mejor calificación ( $p=0.008$ ). Conclusión: Los factores asociados al debut sexual encontrados, fueron en relación a la estructura familiar-social y las actividades en línea, por lo cual es necesario promover programas de educación sexual actualizados.

Palabras Clave: Actividad sexual en línea, debut sexual.
\end{abstract}

\section{ABSTRACT}

Introduction: The sexual practices of adolescents have become a public health problem worldwide. Objective: Describe factors associated with sexual debut in students, online sexual activity and their qualifications. Material and Methods: Observational, cross-sectional study included 673 students from four high schools, 2 public and 2 private in Morelia. A selfadministered survey on factors associated with sexual practices was applied. Results: $27.4 \%$ look for pornography on the internet once a week, cybersex practices $4.5 \%$, sexting $6.1 \%$. Internet was the main source of sexual information (40.4\%). $20.1 \%$ had a sexual debut, with an average of 14.8 years (standard deviation $=1.7$ ), $47.4 \%$ did so out of curiosity. For the sexual debut, family dysfunction and having a partner were significant $(p=0.040$ and $p=0.000)$, the type of institution, religion and self-esteem did not have significance. Students without a sexual debut scored better $(p=0.008)$. Conclusions: The factors found associated with the sexual debut, have relationship to family-social structure and online activities, for this, is necessary to promote updated sex education programs.

Key words: Online sexual activity, sexual debut. 


\section{Introducción}

En la actualidad, el comportamiento sexual de los adolescentes se ha convertido en un problema de salud; ya que las prácticas sexuales se realizan a menor edad, a nivel mundial se estima que la edad promedio de debut sexual va desde los 15 a los 18 años, desencadenando infecciones de transmisión sexual, embarazos no deseados, rezago educativo, abandono escolar, familias disfuncionales, pérdida de productividad profesional, delincuencia, renovación del ciclo de pobreza, complicaciones obstétricas, muertes neonatales, muerte materna y nacimientos pretérmino. (Jarząbek et al 2016; Salam 2016)

Los factores implicados en el cambio de comportamiento sexual, son aquellos que provocan una salida de la neutralidad sexual, generalmente efectos positivos del deseo y la excitación sexual, estos se pueden agrupar en cuatro esferas: biológico (salud física, función hormonal, función genital, función neurobiológica), psicológica (autoestima, personalidad, depresión, ansiedad y consecuencias de abuso sexual), interpersonal (relaciones anteriores, factores estresantes, motivación) y sociocultural (religión, familia, amigos y compañeros, medios de información y medios de comunicación). (Brotto et al 2016; Kingsberg, S. A., Rezaee, R. L. 2013)

Analizando el entorno del adolescente actual, el internet y los avances tecnológicos son los cambios sobresalientes de la época, pudiendo ser los factores de mayor importancia para este cambio de comportamiento sexual.

Mediante el uso de esta tecnología se han creado nuevas actividades sexuales, denominadas actividades sexuales en línea, definidas como el uso de internet para acceder a materiales sexuales como escritura, videos, imágenes, información en general, comprar artículos sexuales, trabajo sexual en internet, ciber sexo y búsqueda de citas o contactos. (Döring 2009; Ucar, Golbasi y Senturk 2016). Se reportan distintos efectos en los usuarios de estas actividades y materiales.

La pornografía, desde el punto de vista psicoanalítico, ayuda a evocar las fantasías sexuales reprimidas. En los niños y adolescentes expuestos a ella puede afectar su desarrollo mental, reflejado en alteraciones de su percepción psicosocial; por lo que se ha recomendado evitar la exposición a este contenido. (Sánchez et al 2007).

El ciber sexo, se practica mediante la estimulación sexual entre personas, a través de mensajes, imágenes y/o video, donde puede o no darse la masturbación. Lo que disminuye los riesgos sexuales físicos, permitiendo gratificación sexual, pero puede generar una conducta adictiva. (Döring 2009)

El sexting, consiste en la difusión o publicación de contenidos como fotografías y videos de tipo sexual, producido por el propio remitente, utilizando para ello cualquier dispositivo tecnológico; es una práctica frecuente entre menores de edad, que por lo general termina afectando al remitente al exponerse de forma pública o en sitios de pornografía infantil. Caso similar con el uso de redes sociales, que se ha convertido en un lugar de captación de contenido, contacto y reclutamiento con propósitos de producción de material pornográfico o explotación. Además de la facilidad para suscribirse a la red y multiplicar contactos que facilitan la actividad sexual. (Ibarra 2014; Delgado y França 2014; Fernández 2013)

Nuestro entorno actual genera personas con un comportamiento inadecuado en uno o varios aspectos de la sexualidad. Por lo cual este trabajo tiene el propósito de mostrar factores relacionados a comportamientos sexuales, incluida la actividad sexual en línea, el debut sexual y su repercusión en la calificación del estudiante, considerando el aspecto académico como parte importante en el desarrollo del adolescente.

\section{Material y Métodos}

Este trabajo se realizó mediante un estudio descriptivo y transversal, donde se encuestaron 673 alumnos de dos escuelas secundarias, una pública y otra privada y dos escuelas preparatorias, de igual manera una pública y otra privada. Se incluyeron estudiantes seleccionados al azar, de ambos sexos y con edad entre 12 y 19 años.

Se aplicó cuestionario diseñado por los autores, donde se incluyeron variables para describir los factores relacionados al comportamiento sexual, incluidos la funcionalidad familiar (prueba de Apgar, 
utilizada por González 2012), nivel de autoestima (Test de Cooper-Smith, utilizada por González 2012) y la actividad sexual en línea. Los cuestionarios se procesaron en el programa estadístico SPSS versión 23, el análisis incluyó medidas de tendencia central, análisis de varianza, de medias e inferencial con los factores asociados, mediante tablas y chi cuadrada. El estudio fue aprobado por el Comité de Ética en Investigación del Hospital (registro HIM-EI17-2016) y de la Facultad (registro CB/2017/V-203). Se obtuvo la aprobación de las instituciones educativas y el asentimiento o consentimiento verbal de los estudiantes para cumplir con el anonimato y confidencialidad.

Variables empleadas en el estudio.

Edad clasificada en grupos de 12 a 15 y 16 a 19 años, debut sexual en años cumplidos, factores asociados al debut sexual en socioculturales (Funcionalidad familiar, influencia de amigos y la religión, relación amorosa y fuente de información sobre sexualidad.), factores psicológicos (Nivel de autoestima) y factores individuales (Motivo para tener la primera relación y debut sexual por violación.) La actividad sexual en línea incluyó búsqueda de pornografía, ciber sexo, sexting y uso de redes sociales para buscar pareja.

\section{Resultados}

La población total del estudio fue de 673 estudiantes, de los cuales $53.6 \%$ (361) fueron del género femenino y el $46.4 \%$ (312) del género masculino. El $58.24 \%$ (392) tenía de 12 a 15 años y $41.76 \%$ de (281) de 16 a 19 años.

El debut sexual se presentó en $20.1 \%$ (135) de los 673 estudiantes, con media de 14.8 años y desviación estándar de 1.7. El debut fue mayor en el grupo de edad de 16 a 19, en el género masculino y tipo de institución privada, la media de edad de debut sexual fue inferior en el grupo de 12 a 15 años ver tabla 1 .

\begin{tabular}{|c|c|c|c|c|}
\hline & \multicolumn{4}{|c|}{ Debut Sexual } \\
\hline & No. & $\%$ & Media & DS \\
\hline \multicolumn{5}{|l|}{ Grupo edad } \\
\hline $12-15$ & 34 & 25.2 & $13.41^{*}$ & 1.2 \\
\hline $16-19$ & 101 & 74.8 & 15.30 & 1.5 \\
\hline \multicolumn{5}{|l|}{ Género } \\
\hline Masculino & 78 & 57.8 & 14.85 & 1.5 \\
\hline Femenino & 57 & 42.2 & 14.79 & 1.9 \\
\hline \multicolumn{5}{|l|}{ Institución } \\
\hline Pública & 50 & 37.0 & 14.84 & 1.8 \\
\hline Privada & 85 & 63.0 & 14.81 & 1.6 \\
\hline General & 135 & 20.1 & 14.80 & 1.7 \\
\hline \multicolumn{5}{|c|}{ Fuente: Directa } \\
\hline${ }^{*} p<.05$ & & & & \\
\hline
\end{tabular}

Tabla 1. Debut sexual por edad, género y tipo de institución

\begin{tabular}{lcccc}
\hline & \multicolumn{4}{c}{ Debut Sexual } \\
& No. & $\%$ & Media & DS \\
\hline $\begin{array}{l}\text { Grupo edad } \\
12-15\end{array}$ & 34 & 25.2 & $13.41^{*}$ & 1.2 \\
$16-19$ & 101 & 74.8 & 15.30 & 1.5 \\
& & & & \\
Género & & & & \\
Masculino & 78 & 57.8 & 14.85 & 1.5 \\
Femenino & 57 & 42.2 & 14.79 & 1.9 \\
& & & & \\
Institución & & & & \\
Pública & 50 & 37.0 & 14.84 & 1.8 \\
Privada & 85 & 63.0 & 14.81 & 1.6 \\
General & 135 & 20.1 & 14.80 & 1.7 \\
\hline
\end{tabular}

Fuente: Directa

$* p<.05$

En cuanto a la actividad sexual en línea, la búsqueda de pornografía fue la principal actividad (27.4\%), donde en todas las actividades, los hombres de institución privada fueron los de mayor frecuencia 
con significancia estadística $(\mathrm{p}=0.000$ y $\mathrm{p}=0.004)$ (ver tabla 2).

Tabla 2. Prácticas de la actividad sexual en línea

\begin{tabular}{|c|c|c|c|c|c|c|c|c|c|c|}
\hline \multirow[b]{2}{*}{ Tipo de actividad } & \multirow[b]{2}{*}{ No. } & \multirow[b]{2}{*}{$\%$} & \multicolumn{2}{|c|}{ Hombres } & \multicolumn{2}{|c|}{ Mujeres } & \multicolumn{2}{|c|}{ Inst. Pública } & \multicolumn{2}{|c|}{ Inst. Privada } \\
\hline & & & No. & $\%$ & No. & $\%$ & No. & $\%$ & No. & $\%$ \\
\hline \multicolumn{11}{|l|}{ Búsqueda de } \\
\hline pornografía & 184.0 & 27.4 & 152.0 & 82.0 & 32.0 & 18.0 & 51.0 & 28.0 & 133.0 & 72.0 \\
\hline Ciber sexo & 30.0 & 4.5 & 20.0 & 67.0 & 10.0 & 33.0 & 6.0 & 20.0 & 24.0 & 80.0 \\
\hline Sexting & 41.0 & 6.1 & 24.0 & 58.0 & 17.0 & 42.0 & 8.0 & 20.0 & 33.0 & 80.0 \\
\hline \multicolumn{11}{|l|}{$\begin{array}{l}\text { Busqueda de } \\
\text { pareja sexual o }\end{array}$} \\
\hline romántica & 40.0 & 5.9 & 29.0 & 73.0 & 11.0 & 27.0 & 15.0 & 38.0 & 25.0 & 62.0 \\
\hline
\end{tabular}

La frecuencia más común de búsqueda de pornografía es 1 a 2 veces por semana (20.4\%), seguida de 3 a 4 veces (3.1\%), 5 a 7 veces $(2.7 \%)$ y por ultimo más de una vez al día (1.2\%). Todas las actividades sexuales en línea resultaron estadísticamente significativas al asociarla con el debut sexual $(\mathrm{p}=0.000)$. El tiempo dedicado para buscar pareja en redes sociales obtuvo una media de 1.9 horas al día, con desviación estándar de 1.3 horas y rango de 5.

La descripción de los factores del debut sexual (tabla 3 ), mostró que la mayor parte de los estudiantes tienen una familia funcional (66.7\%), sin embargo, resulto significativo la disfunción familiar para el debut sexual $(\mathrm{p}=0.04)$. El $79.4 \%$ indico pertenecer a la religión católica, seguido del $13.9 \%$ sin religión, sin tener significancia para el debut sexual $(\mathrm{p}=0.077)$. El internet fue la fuente de información más mencionada por los encuestados (40.4\%), sin ser significante para el debut sexual ( $\mathrm{p}=0.155)$. El 53.6\% de los estudiantes tienen una tendencia a alta autoestima, seguido del $21.7 \%$ con tendencia a baja autoestima, sin significancia $(\mathrm{p}=0.879)$. La curiosidad fue el principal motivo para el debut sexual (47.4\%) y en tres estudiantes de preparatoria privada su debut fue producto de violación. El 62.1\% de los estudiantes cuentan con una relación amorosa, resultando significativo para el debut sexual $(p=0.000)$. Cuestionando sobre el tiempo dedicado a la pareja, tanto en la relación personal como en la comunicación digital, tuvo una media de 4.6 horas al día, desviación estándar de 2.99 horas, un valor mínimo de 1 hora y máximo de 13.

$\mathrm{Al}$ cuestionar sobre la persona con la cual se tuvo el debut sexual, la pareja fue la más mencionada (56.3\%), seguido de un amigo/amiga (25.2\%) y
$12.7 \%$ no especificaron con quien tuvo su primera relación.

Se encontró una media de calificación de 8.03 con una desviación estándar de 0.978 puntos. Dos estudiantes tienen calificación reprobatoria y 29 excelentes. Al clasificar las calificaciones se agruparon en competentes ( 8 a 10) correspondiendo a este grupo un $72.8 \%$ (490) y no competente ( 5 a 7 ) que obtuvo el porcentaje restante. La media de calificación del grupo de estudiantes sin debut sexual fue de 8.08 (desviación estándar=0.95), superior al 7.81 (desviación estándar=1.05) del grupo con debut sexual. La calificación resultó estar asociada al debut sexual, al aplicar la prueba de Chi-cuadrada $(\mathrm{p}=0.008)$ y fue mejor en el grupo de estudiantes $\sin$ debut sexual, (ANOVA p=0.004). (tabla 4).

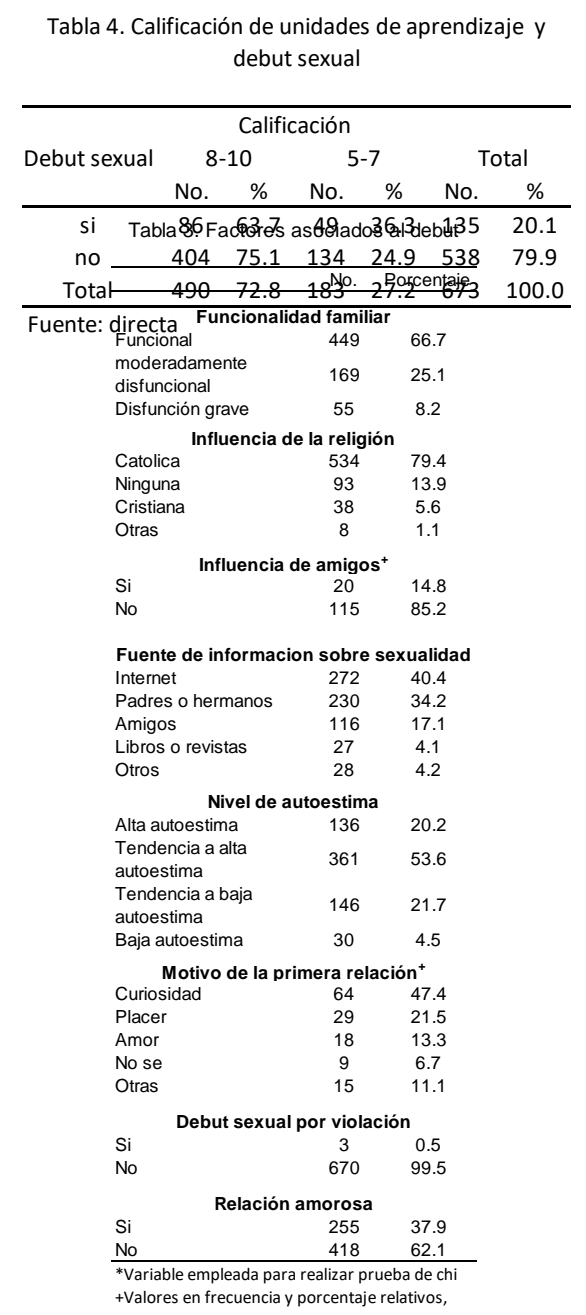




\section{Discusión}

Obtuvimos una media de edad de debut sexual de 14.8 años, inferior al 16.5 reportado por Fernández 2013 y coincidente con el 14.5 de Carvajal 2017 en Colombia, esta similitud podría deberse a que, en la actualidad, el inicio de las relaciones sexuales en los alumnos de secundaria y preparatoria, ocurre en edades más tempranas, al tener mayor información y tiempo para la sexualidad. Así lo muestran estudios poblacionales, como el realizado en EUA por National Center for Health Statistics 2015, que reporta una edad de debut sexual de 17.3 años para las mujeres y 17 en los hombres, que es notablemente superior (2.5 años) a los 14.8 obtenido en nuestro estudio. Probablemente debido a que corresponde a un estudio poblacional y a las diferencias sociales y culturales que se tienen con ese país.

El Consejo Nacional de Población reporta que en Michoacán (CONAPO 2011), la media de edad de debut sexual es 18.2 años, que es 3.4 años mayor al nuestro, probablemente debido a que estudiamos adolescentes y no población general.

El debut sexual en los hombres tuvo diferencias significativas al de las mujeres (25\% vs $16 \%)$, coincidentes con el de Fernández 2017 (77.0\% vs $66.0 \%$ ), estas diferencias fueron menores conforme aumenta la edad, como lo reporta el estudio de Leyva 2010, al no encontrar significancia a 18 y 19 años, probablemente intervenga la política de equidad de género.

El debut sexual fue más frecuente con la pareja (56.3\%), lo que coincide con Leyva 2010, aunque con un porcentaje mayor $(89.4 \%)$, el debut con amigo fue mayor al nuestro $(25.2 \%$ vs $18.63 \%)$ y el debut con otras personas fue más frecuente en nuestro estudio ( $18.5 \%$ vs $4.23 \%$ ); probablemente debido a la tecnología de la información que permiten informarse y experimentar sexualidad de distinta forma. También coincidimos con Leyva 2010 sobre los principales motivos de debut sexual (curiosidad, placer y amor).

Sobre búsqueda de pornografía, Álvarez 2008 en Perú señala que $30.4 \%$ de estudiantes de 10 a 19 años la realiza, porcentaje similar al nuestro $(27.4 \%)$. En cambio, Vizcarral 2004, en estudiantes chilenos de
11 a 12 años, el porcentaje es $51 \%$, lo que podría deberse a características sociales de cada país, cuya alta frecuencia requiere mayor atención a este grupo etario considerando que al aumentar la edad y nivel educativo la búsqueda se incrementa hasta $96 \%$ reportado por Boies 2002 en universitarios canadienses.

El sexting tiene frecuencia variable en población joven, en la revisión de Sanllehí 2017 reporta rango de $2.5 \%$ a $30 \%$. El $6.1 \%$ de nuestro estudio está dentro de este rango, siendo más coincidente al 7.9\%, reportado por Organización Efecto Internet (2017) en 10,000 adolescentes mexicanos; la diferencia puede deberse a que el estudio de Sanllehí incluye poblaciones de diferentes países.

El 25.6\% de universitarios canadienses practica ciber sexo (Boies 2002), 9.3\% en estudiantes de colegios koreanos (Park 2013). Frecuencias superiores al $4.5 \%$ de nuestro estudio; esto podría deberse a la edad mayor de universitarios canadienses y que nuestra frecuencia sea más cercana a los colegios Koreanos.

La significancia estadística obtenida entre el debut sexual y las actividades sexuales en línea, se interpretó como que los estudiantes con debut sexual practican este tipo de actividades, ya que no fue tan frecuente para asociarla directamente al debut sexual. La disfunción familiar en nuestro estudio, fue una variable estadísticamente significativa para el debut sexual, que difiere de la no significancia reportada por González 2012, lo cual puede deberse a diferencias en el tamaño de población estudiada y año realizado.

En la literatura revisada no se encontró información sobre la repercusión del debut sexual en la calificación de los estudiantes. Nosotros abordamos este aspecto y detectamos que hubo mejor calificación en los estudiantes sin debut sexual, diferencia que resultó estadísticamente significativa, lo que sugiere que el no haber tenido relaciones sexuales, puede favorecer un mayor tiempo para el estudio y mejor nivel de calificación, aunque hay que tomar en cuenta que en la calificación influyen otros factores que no se estudiaron.

\section{Conclusiones}


Los resultados de este trabajo apoyan la necesidad de promover en escuelas de nivel medio programas actualizados de educación sexual, tomando en cuenta que los factores relacionados al debut sexual están en relación a la estructura familiar y las actividades en línea, para fortalecer el ejercicio responsable de la misma, sensibilizar a alumnos y padres, sobre los riesgos que representa el abuso del acceso a internet; además de promover líneas de investigación sobre la vida sexual de los adolescentes e intervenciones que disminuyan el comportamiento de riesgo.

La calificación mayor en los estudiantes sin debut sexual pudiera ser una coincidencia, por lo cual se requieren más estudios al respecto.

\section{Agradecimientos:}

Los autores agradecen al personal docente y alumnos de las instituciones educativas por su autorización y participación. Al departamento de enseñanza e investigación por apoyo y asesoría metodológica.

\section{Bibliografía}

Alvarez, J. A. S. M., Urdanivia, K. J. L., Romero, L. D. R. E., Zare, C. T. Z., Rengifo, D. L., Irala, C. E., \& Granara, A. S. (2008). Acceso a páginas pornográficas en Internet y Comunicación familiar sobre sexualidad en adolescentes del distrito de" El Agustino", LimaPerú 2006-2007. Horizonte Médico, 8(1). Recuperado de:

http://www.horizontemedicina.usmp.edu.pe/index.ph p/horizontemed/article/view/194

Boies, S. C. (2002). University students' uses of and reactions to online sexual information and entertainment: Links to online and offline sexual behaviour. The Canadian Journal of Human Sexuality, 11(2), $77 . \quad$ Recuperado de: https://search.proquest.com/openview/3b9779765e08 e9c3198e3dba85050a40/1?pq$\underline{\text { origsite }=\text { gscholar } \& \mathrm{cbl}=33400}$

Brotto, L., Atallah, S., Johnson-Agbakwu, C., Rosenbaum, T., Abdo, C., Byers, E. S., ... \& Wylie, K. (2016). Psychological and interpersonal dimensions of sexual function and dysfunction. The journal of sexual medicine, 13(4), 538-571. Recuperado de: https://www.sciencedirect.com/science/article/pii/S17 43609516003052

Carvajal Barona, R., Oliveros, V., Leder, H., \& Rodríguez Amaya, R. M. (2017). Factores asociados al embarazo en adolescentes de 13 a 19 años en el municipio de
Buenaventura, Colombia. Revista de la Universidad Industrial de Santander. Salud, 49(2), 290-300. Recuperado de: http://www.scielo.org.co/scielo.php?script=sci_abstra ct\&pid=S0121-08072017000200290

CONAPO. Consejo Nacional de Población. (2011) Perfiles de Salud Reproductiva Michoacán. Paloma Villagómez Ornelas, Doroteo Mendoza Victorino, Jorge Armando Valencia Rodríguez. Recuperado de: https://www.gob.mx/conapo/documentos/perfiles-desalud-reproductiva-2011

Delgado Coto, S., \& França Tarragó, O. (2014). FLUJO DE MATERIAL PORNOGRÁFICO INFANTIL ONLINE: ESTUDIO EXPLORATORIO EN 10 PAÍSES DE AMÉRICA LATINA CON FOCO EN URUGUAY. Ciencias Psicológicas, 8(1), 55-67. Recuperado de: http://www.scielo.edu.uy/scielo.php?pid=S1688$\underline{42212014000100006 \& \text { script }=\text { sci_arttext\&tlng=es }}$

Döring, N. M. (2009). The Internet's impact on sexuality: A critical review of 15 years of research. Computers in Human Behavior, 25(5), 1089-1101. Recuperado de: https://www.sciencedirect.com/science/article/pii/S07 47563209000442

El efecto Internet (www.asi-mexico.org). (2017) Sexting, Cuando la diversión se convierte en delito. Año 2, número 2. Recuperado de: http: //asimexico.org/sitio/archivos/Revista_baja_Sexting_ $\overline{5 . p d f}$

Fernández Cáceres, C., Rodríguez Kuri, S. E., Pérez Islas, V., \& Alcaráz, C. (2017). Prácticas sexuales de riesgo y su relación con el consumo de alcohol y otras drogas en estudiantes de educación media y superior. Revista Médica de la Universidad Veracruzana, 16(1), 19-29. Recuperado de: http://www.medigraphic.com/cgibin/new/resumen.cgi?IDARTICULO=70771

Fernández Sánchez, N. (2013). Trastornos de conducta y redes sociales en Internet. Salud mental, 36(6), 521$527 . \quad$ Recuperado de: http://www.scielo.org.mx/scielo.php?pid=S018533252013000600010\&script=sci_arttext

González-Quiñones, J. C., Salamanca-Preciado, J. P., Quiroz-Rivera, R. M., Hernández-Pardo, Á. M., Hernández-Rojas, A. D., \& Quesada-Núñez, B. (2012). Identificación de factores de riesgo de embarazo en población adolescente escolar urbana y rural colombiana. Rev. salud pública, 14(3), 404-16. Recuperado

de: https://www.scielosp.org/article/ssm/content/raw/?res ource_ssm_path=/media/assets/rsap/v14n3/v14n3a04. pdf 
Ibarra Sánchez, E. (2014). Protección de niños en la red: Sexting, ciberbullying y pornografía infantil. I. Luna Pla, Estudios aplicados sobre la libertad de expresión y el derecho a la información, 83-115. Recuperado de: https://archivos.juridicas.unam.mx/www/bjv/libros/8/ $\underline{3646 / 5 . p d f}$

Instituto Nacional de Atención a las Mujeres. (2016). Estrategia Nacional para la Prevención del Embarazo en Adolescentes. Recuperada de: http://www.gob.mx/inmujeres/acciones-yprogramas/estrategia-nacional-para-la-prevenciondel-embarazo-en-adolescentes-33454

Jarząbek-Bielecka, G., Pisarska-Krawczyk, M., Mizgier, M., Andrzejak, K., Sajdak, S., \& Kędzia, W. (2016) Preventing sexually transmitted diseases (including HPV and HIV) in the context of premature sexual initiation, health promotion, and other related issues for gynaecological practice-a review. Journal of Health Inequalities, 2(2), 200-204. Recuperada de: https://www.termedia.pl/,100,29233,0,1.html

Kingsberg, S. A., \& Rezaee, R. L. (2013). Hypoactive sexual desire in women. Menopause, 20(12), 1284$1300 . \quad$ Recuperada de: https://journals.1ww.com/menopausejournal/Abstract/ 2013/12000/Hypoactive_sexual_desire_in_women.12 aspx

Leyva-López, Leyva-López, A., Chávez-Ayala, R., Atienzo, E. E., Allen-Leigh, B., Ramírez-Villalobos, D., Yunes-Díaz, E., \& Rivera-Rivera, L. (2010). Anticoncepción de emergencia en estudiantes mexicanos. salud pública de méxico, 52, 156-164. Recuperada de: https://www.scielosp.org/scielo.php?pid=S0036$\underline{36342010000200008 \& \text { script }=\text { sci_arttext\&tlng }=}$

National Center for Health Statistics. (2015) Key Statistics from the National Survey of Family Growth. Recuperado de: http://www.cdc.gov/nchs/nsfg/key_statistics/s.htm\#se $\underline{\text { xualactivity }}$

Salam, R. A., Faqqah, A., Sajjad, N., Lassi, Z. S., Das, J. K., Kaufman, M., \& Bhutta, Z. A. (2016). Improving adolescent sexual and reproductive health: A systematic review of potential interventions. Journal of Adolescent Health, 59(4), S11-S28. Recuperado de: https://www.jahonline.org/article/S1054$\underline{139 X(16) 30168-9 / \text { fulltext }}$

Sánchez-Chávez, N. P., Reyes-Hernández, U., ReyesHernández, D., Quero-Hernández, A., Reyes-Gómez, U., \& Colón-Cuesta, F. (2007). Entorno de la Consulta de Pornografía y su repercusión en Relación a la Sexualidad en un Grupo de Adolescentes Masculinos. Boletín Clínico Hospital Infantil del Estado de Sonora, 24(1), 3-8. Recuperado de: http://www.medigraphic.com/pdfs/bolclinhosinfson/bi s-2007/bis071b.pdf

Sanllehí, J. R. A., \& Montiel, I. (2017). Sexting en adolescentes: nuevos retos médico-legales. Revista española de medicina legal: órgano de la Asociación Nacional de Médicos Forenses, 43(1), 43-44. Recuperado de: http://www.elsevier.es/es-revistarevista-espanola-medicina-legal-285-articulo-sextingadolescentes-nuevos-retos-medico-legales$\underline{\mathrm{S} 0377473216300487}$

Park, H., \& Kang, S. J. (2013). Cybersex addiction among Korean college students: current status and relationships of sexual knowledge and sexual attitude. Journal of Korean Public Health Nursing, 27(3), 608$618 . \quad$ Recuperado de: http://www.koreascience.or.kr/article/ArticleFullReco rd.jsp?cn=BGGHB8_2013_v27n3_608

Ucar, T., Golbasi, Z., \& Senturk Erenel, A. (2016). Sexuality and the internet: a study of the perspectives of Turkish university students. Cyberpsychology, Behavior, and Social Networking, 19(12), 740-745. Recuperado de: https://www.liebertpub.com/doi/abs/10.1089/cyber.20 $\underline{16.0433}$

Vizcarral, M. B., Balladares, E., Candia, C., Lepe, M., \& Saldivia, C. (2004). Conducta sexual durante la infancia en estudiantes chilenos. Psicothema, 16(1). Recuperado de: http://www.redalyc.org/html/727/72716110/ 